International Conference Mathematical and Computational Biology 2011

International Journal of Modern Physics: Conference Series

Vol. 9 (2012) 406-411

(C) World Scientific Publishing Company

DOI: $10.1142 / \mathrm{S} 201019451200548 \mathrm{X}$

\title{
LOADING CONTAINERS WITH ANTS
}

\author{
CHING NEI YAP \\ Laboratory of Computational Statistics and Operations Research, Institute for Mathematical Research, \\ Universiti Putra Malaysia, 43400 UPM Serdang, Selangor Darul Ehsan, Malaysia \\ yapjuny@gmail.com \\ LAI SOON LEE \\ Laboratory of Computational Statistics and Operations Research, Institute for Mathematical Research, \\ Department of Mathematics, Faculty of Science, \\ Universiti Putra Malaysia, 43400 UPM Serdang, Selangor Darul Ehsan, Malaysia \\ lee@math.upm.edu.my \\ ZANARIAH ABDUL MAJID \\ Laboratory of Computational Sciences and Mathematical Physics, Institute for Mathematical Research, \\ Department of Mathematics, Faculty of Science, \\ Universiti Putra Malaysia, 43400 UPM Serdang, Selangor Darul Ehsan, Malaysia \\ zanariah@math.upm.edu.my \\ HSIN VONN SEOW \\ Nottingham University Business School, University of Nottingham Malaysia Campus, \\ Jalan Broga, 43500 Semenyih, Selangor Darul Ehsan, Malaysia \\ Hsin-Vonn.Seow@nottingham.edu.my
}

\begin{abstract}
Loading containers is like loading a subset of given three-dimensional rectangular boxes of different sizes into a three-dimensional rectangular container of fixed dimensions in order to achieve optimal space utilization. In this paper, Ant Colony Optimization (ACO) with its probabilistic decision rule is used to construct towers of boxes and to arrange them into the container. Some initial computational results on benchmark data set will be presented.
\end{abstract}

Keywords: Loading containers; space utilization; ant colony optimization.

\section{Introduction}

Loading containers involve packing a subset of given rectangular boxes into a rectangular container of fixed dimensions and is called Container Loading Problem (CLP). CLP belongs to the combinatorial optimization problems which focuses on achieving an optimal space utilization given a limited space. This problem has numerous applications in the cutting and packing industry, pallets loading or container utilization in the logistics industry. Filling containers in an optimal way can reduce shipping costs. The CLP can be defined as 
"Given a set of $n$ three-dimensional rectangular box types $j \in$ $\{1,2, \ldots, n\}$, each is given height $h_{j}$, width $w_{j}$, depth $d_{j}$ and quantity $m_{j}$, and a three-dimensional rectangular container with height $H$, width $W$ and depth $D$. The objective is to pack a subset of the given boxes into the container in such a way that is optimal in terms of the available container space, and additional constraints must be met where necessary."

The CLP can be classified into three categories based on the number of different box types. These are the homogeneous problem with one type of box only, the weakly heterogeneous problem with relatively few box types and many boxes of each type, and the strongly heterogeneous problem with many different types of boxes and few boxes of each type.

In this paper, Ant Colony Optimization (ACO) has been used to solve CLP. We use ACO probabilistic rule and pheromone update to find the optimal solution.

The remainder of this paper is organized as follows: literature review will be covered in Section 2. Section 3 describes the application of ACO in CLP. Some computational experiments are documented in Section 4 and finally, a conclusion and some future works will be explained in Section 5.

\section{Literature Review}

CLP has already been solved by many scholars with different methods. The stack building approach is proposed by Gilmore and Gomory ${ }^{1}$, which solved the problem by packing the boxes into suitable stacks then are arranged on the floor of the container by solving a two-dimensional packing problem. Gehring and Bortfeldt ${ }^{2}$ presented a heuristic based on this idea by using Genetic Algorithm (GA) to solve the two-dimensional packing problem. ${ }^{3}$

Ant Colony Optimization (ACO) was introduced by Dorigo and Stützle ${ }^{4}$, which is a population-based meta-heuristic for hard combinatorial optimization problem. It is inspired by the behavior of colonies of real ants in searching for food from their nests because most of the ants use the shortest route from the nest to the food source after a few transitory phases. This is because of the indirect communication among the individual "artificial ants" using pheromones which real ants use for communication. At each step of constructing a solution, the ants select the best feasible arc according to a probabilistic rule which is based on the heuristic information $\eta_{i j}$ and pheromone trail level $\tau_{i j}$ of each $\operatorname{arc}(i, j)$. The higher the heuristic value and pheromone trail of the arc, the higher the probability it will be selected. The heuristic value represents a priori information about the problem or run time information provided by a source different from the ants. The amount (intensity) of "artificial pheromone" trail is proportional to the utility of the arc which is used to build the solution and is estimated by the ants. Pheromone evaporation will help to avoid a too rapid convergence of the algorithm towards a sub-optimal region. It is also a form of forgetting, favoring the exploration of new areas of the search space. 
Liang et al. ${ }^{5}$ proposed a hybrid meta-heuristic with a two-phased method based on GA and ACO to solve the CLP. In the first phase, the pheromone updating mechanism in ACO is used to choose the proper box and the decision rule is used to construct tower set. In the second phase, GA is used to arrange the towers on the container's bottom plane. Besides that, ACO for complex CLP is studied by Zhuang et al. ${ }^{6}$.

\section{ACO for CLP}

In this section, the application of ACO in CLP will be described. The main focus is to find the best Space Utilization Ratio (SUR). CLP can be formulated as:

$$
\max S U R=\frac{\sum_{r=1}^{v} V_{r}}{C}
$$

where $v=$ quantity of already packed box

$V_{r}=$ volume of the $r_{t h}$ packed box

$\mathrm{C}=$ volume of the container

The procedure of the application of ACO in CLP can be summarized as:

$$
\text { procedure ACO for CLP }
$$

begin

Initialize pheromone

repeat

for each ant do

Randomly choose a box as the first box

Choose next box by applying the probability function $P_{i j}$

Until the ant has built a sequence of boxes

Pack the boxes into container according to the sequence until container is full

Apply pheromone update

end for

Choose the best solution as our solution

end

\subsection{Probability function}

The procedure begins with the initialization of the pheromone trails, the ants construct the sequence of boxes starting with a random box. At each step, the ant apply the probability

$$
P_{i j}=\frac{\tau_{i j \times \eta_{i j}}}{\sum_{k \in D}\left(\tau_{i k} \times \eta_{i k}\right)} \text {, if } j \in D
$$

where $\tau_{i j}=$ pheromone trails

$\eta_{i j}=$ the bottom area of box $j$

to choose box $j \in D$ as the next box when the current box is box $i$, where $D$ is the set of boxes that have not been chosen yet. 


\subsection{Heuristic approach}

The stack building approach which is proposed by Gilmore and Gomory ${ }^{1}$ is used to pack the boxes into the container. The boxes are packed by stacking them one by one according to the sequence until the container is full. The SUR is then calculated according to Eq. (1).

\subsection{Pheromone update}

After the container is full, the pheromone of the boxes that have been packed in the container will be updated by

$$
\tau_{i j}=(1-\rho) \tau_{i j}+S U R
$$

where $\rho=(0,1]$ : evaporation rate

The aim of the pheromone evaporation rate is to reduce the chance of the other ants selecting the same solution and consequently the search will be more diversified. The CLP solution will be represented by the best SUR.

\section{Computational Experiments}

The experiments are solved by using $\mathrm{C}$ programming and tested on five problem instances in benchmark datasets of three box types. The datasets were generated and used by Bischoff and Ratcliff $^{7}$ and which can be downloaded from http://people.brunel.ac.uk/ mastijb/jeb/info.html.

Table 1 shows the computational results and are tested on different evaporation rates, $\rho$. Column (a) represents the results of using Eq. (4) as the probability to choose the next box which is proposed by Liang et al. ${ }^{5}$ and column (b) represents our results using the proposed probability in Eq. (2). Each problem is tested five times and the results showed are an average value of the percentage of SUR value in Eq. (1). On average, our proposed method has better results.

$$
P_{i j}=\frac{\tau_{i j+}+\eta_{i j}}{\sum_{k \in D}\left(\tau_{i k}+\eta_{i k}\right)}, \text { if } j \in D
$$

\section{Conclusion}

This paper solved the CLP by applying ACO. The probabilistic rule and the pheromone feedback were used to construct the solution. We observed from the experimental results, the proposed probability function can generally yield better results.

The future works will see ACO usage be extended to work on more box types problems. 
Table 1. Computational results.

\begin{tabular}{llll}
\hline Prob Instance & $\rho$ & $(\mathrm{a})$ & $(\mathrm{b})$ \\
\hline 1 & 0.1 & 85.86 & 86.36 \\
& 0.3 & 85.86 & 86.85 \\
& 0.5 & 85.86 & 86.33 \\
& 0.7 & 85.86 & 88.52 \\
& 0.9 & 85.86 & 86.65 \\
\hline 2 & 0.1 & 82.43 & 83.29 \\
& 0.3 & 82.43 & 83.56 \\
& 0.5 & 82.43 & 83.30 \\
& 0.7 & 82.43 & 82.43 \\
& 0.9 & 82.43 & 82.43 \\
\hline 4 & 0.1 & 66.49 & 63.11 \\
& 0.3 & 66.49 & 64.24 \\
& 0.5 & 66.49 & 66.49 \\
& 0.7 & 66.49 & 65.37 \\
& 0.9 & 66.49 & 63.82 \\
\hline 5 & 0.1 & 77.40 & 78.29 \\
& 0.3 & 77.40 & 77.40 \\
& 0.5 & 77.40 & 77.40 \\
& 0.7 & 77.40 & 77.40 \\
& 0.9 & 77.40 & 77.40 \\
\hline & 0.1 & 82.24 & 83.59 \\
& 0.3 & 82.24 & 86.25 \\
& 0.5 & 82.24 & 86.11 \\
& 0.7 & 82.24 & 85.21 \\
& 0.9 & 82.24 & 83.74 \\
\hline
\end{tabular}

\section{References}

1. P. C. Gilmore and R. E. Gomory, Multistage Cutting Stock Problems of two and more dimensions, Operations Research, 13, 94-120, (1965).

2. H. Gehring and A. Bortfeldt, A Genetic Algorithm for solving the Container Loading Problem, International Transactions in Operational Research, 4, 401-418, (1997).

3. D. Pisinger, Heuristics for the Container Loading Problem, European Journal of Operational Resesarch, 141, 382-392, (2002).

4. M. Dorigo and T. Stützle (eds.), Ant Colony Optimization. (The MIT Press, Cambridge, MA, 2004).

5. S. C. Liang, C. Y. Lee and S. W. Huang, A Hybrid Meta-heuristic for the Container Loading Problem, Communications of the IIMA, Volume 7 Issue 4, 73-84, (2007).

6. F. T. Zhuang, L. Zhang, C. X. Zhang and S. Gao, Research on solution to Container Loading Problem based on Ant Colony Optimization, Journal of Jiangnan University (Natural Science Edition), Volume 6 No. 6, 795-799, (2007).

7. E.E. Bischoff and M.S.W. Ratcliff, Issues in the development of Approaches to Container Loading, Omega International Journal Management Science, Volume 23 No. 4, 377-390, (1995). 